\title{
Editorial
}

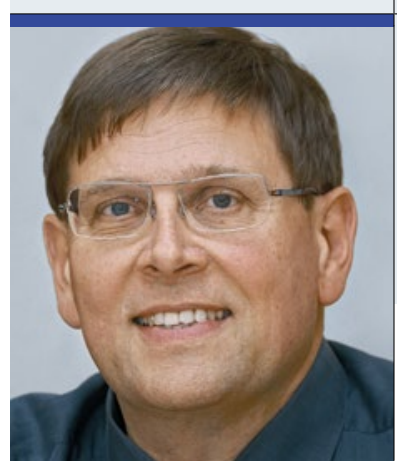

"Eine Bedarfsplanung oder Sicherstellung der Versorgung von

Patienten mit schweren Schmerzen existiert auch heute noch in

keiner Kassenärztlichen Vereinigung."

Dr. med. Gerhard H. H. Müller-Schwefe, Göppingen

Präsident der Deutschen Gesellschaft für Schmerzmedizin e.V.

\section{Aufbruch in eine neue Versorgungswelt}

$\mathrm{N}$ ichts ist besser geworden, Schmerzpatienten sind heute schlechter versorgt als vor zwei Jahren, ihre Zahl hat zugenommen auf inzwischen 3,4 Millionen Menschen mit schwersten problematischen Schmerzen“, klagte Michael A. Überall, Präsident der Deutschen Schmerzliga, auf dem 2. Nationalen Versorgungsforum Schmerz im März 2017 in Berlin. Susanne Wüste, Vizepräsidenten der Deutschen Schmerzliga kommentierte: „Schmerzpatienten müssen teilweise über $100 \mathrm{~km}$ weit fahren, um einen kompetenten Ansprechpartner zu finden " und Joachim Nadstawek, Präsident des BVSD, forderte „eine Schutzzone für Schmerzmediziner", um wenigstens einen Bestandschutz für existierende Einrichtungen zu ermöglichen, da immer weniger Schmerzmediziner Nachfolger für ihre Praxen finden können.

Vertreter von Krankenkassen, Kassenärztlicher Bundesvereinigung, Physiotherapeuten, Psychotherapeuten, Apothekern sowie Politik und Fachgesellschaften waren der Einladung von DSL, DGS und BVSD gefolgt, um nach zwei Jahren die Versorgungssituation von Patienten mit chronischen Schmerzen erneut zu analysieren. Die von großer Offenheit aller Beteiligten geprägte Diskussion bestätigte abermals, dass die Versorgungssituation für die meisten Betroffenen weiterhin unerträglich ist, dass - wie schon vor zwei Jahren - auch heute noch eine Bedarfsplanung oder Sicherstellung der Versorgung in keiner Kassenärztlichen Vereinigung existiert. Das von den Veranstaltern weiterhin für richtig gehaltene Konzept eines Querschnittsfaches "Schmerzmedizin“ mit einem entsprechenden Facharzt würde zwar die Möglichkeit zu einer fachgebietsorientierten Bedarfsplanung eröffnen, scheitert aber weiterhin an den Widerständen zahlreicher Fachgesellschaften. Unter diesem Aspekt war sich die Konferenz einig, dass neue Versorgungskonzepte einen Weg aus dem Dilemma weisen könnten.
Das Commitment aller Beteiligten, konstruktiv an einem solchen Konzept mitarbeiten zu wollen, eröffnet Perspektiven für eine bessere Versorgung in der Zukunft. Einen ersten Entwurf dieses Konzeptes finden Sie ab Seite 38.

Wieder einmal bildet diese Ausgabe die ganze Themenvielfalt der Schmerz- und Palliativmedizin ab. Ganz besonders ans Herz legen möchte ich Ihnen jedoch die Berichterstattung vom Deutschen Schmerz- und Palliativtag 2017, der sich intensiv mit dem Thema Schmerzversorgung in Theorie und Praxis beschäftigt hat (ab Seite 10), sowie die Arbeit von Hanne Albert, der Preisträgerin des Deutschen Schmerzpreises 2017 (Seite 40). Über ihre revolutionären Ansätze der Behandlung von chronischen Rückenschmerzen mit Antibiotika konnten Sie bereits in der letzten Ausgabe dieser Zeitschrift lesen. In zahlreichen Diskussionsforen habe ich herablassende und mitleidige Kommentare zu dieser Thematik gefunden. Eine seriöse Auseinandersetzung mit den Daten lohnt sich und könnte auch ihr Weltbild verändern.

Ich wünsche Ihnen viel Freude mit dieser Ausgabe der "Schmerzmedizin - Angewandte Schmerztherapie und Palliativmedizin" und verbleibe mit herzlichen Grüßen,

Ihr

Gerhard H. H. Müller-Schwefe 